BULL. AUSTRAL. MATH. SOC.

VOL. 34 (1986) 11-23.

\title{
POLYNOMIAL RINGS OVER RINGS INTEGRAL OVER THEIR CENTRES
}

\author{
C.L. WANGNeo
}

\begin{abstract}
We prove in this paper that every finitely generated critical module over $A[X]$ is compressible where $A$ is a Noetherian ring integral over a subring of its centre. Here $A[X]$ denotes the polynomial ring over $A$ in a commuting indeterminate $X$.
\end{abstract}

Introduction

This paper is divided into two sections. Section 1 is essentially devoted to extending results of Wangneo and Tewari ([14] Section 2) to arbitrary right Noetherian rings. We define the support of a finitely generated module $M$ over a right Noetherian ring $B$ and show that if a prime $P$ is a minimal element of the support of $M$ then a prime ideal $Q$ is associated with $M$ if $Q$ lies over $P \cap R$ where $R$ is any subring of the centre of $B$ containing its identity. This result may be used to determine the assassinators of finitely generated modules over right Noetherian rings which are well behaved with respect to their centres. In the present paper we use it to prove in Section 2 that finitely generated critical modules over $A[X]$ are compressible where $A$ is a two-sided Noetherian ring integral over a subring of its centre. This is proved in the uncertainty of fully bounded Noetherianness of $A[X]$. Note that Jategaonker [9] has proved that over a fully bounded Noetherian ring, every finitely generated critical module is compressible. However proving $A[X]$ is fully bounded Noetherian, even in the case when $A$ is a division ring algebraic over its centre, is another version of an open problem due to Amitsur [1].

Received 3 september 1985. The author is indebted to Professor Ann K. Boyle for going through a preliminary version of the paper.

Copyright Clearance Centre, Inc. Serial-fee code: 0004-9727/86 $\$ A 2.00+0.00$. 
Also note that in general setting Goodearl and Lenagen ([8], p. 863) have given an example of a right and left Noetherian ring $Q$ such that all finitely generated critical $Q$-modules are compressible but such that the polynomial ring $Q[X]$ possesses incompressible finitely generated critical modules.

Finally we conclude this introduction with a few remarks conceming our notation and terminology. All our basic definitions and terminology are given in Section $I$ of wangneo and Tewari [14] except that whenever we refer to a ring $A$ we do not necessarily mean (unless otherwise stated) that $A$ is a right Noetherian ring integral over a subring $R$ of its centre. If $B$ is a ring, then $B[X]$ will mean the polynomial ring over $B$ in a comuting variable $X$; the centre of $B$ will be denoted by $Z(B)$ and all rings mentioned in this paper possess an identity. Also, if $M$ is a right $B$-module, then $E_{B}(M)$ will denote the injective hull of $M$ over $B$.

1.

We begin by extending results of Wangneo and Tewari ([14] Section 2) to any right Noetherian ring. We also prove some preliminary results about polynomial rings over right Noetherian rings integral over their centres which will be used later in Section 2 .

PROPOSITION 1.1. Let $A$ be a right lloetherion ring. Then for every cyclic, prime, critical module $M$ with annihilator $P$ and $M \approx \frac{A}{I}$ (I a right ideal of $A$ ) we have:

either (i) $M$ is admissible in which case $P$, the conihizator of $M$ is a completely prime ideal of $A$,

or (ii) $I$ is a prime right ideal of $A$ and $P$ is the bound of $I$ in $A$.

Proof. If $M$ is admissible, then $I=P$ and so $A / I$ is a prime ring which is also uniform as a right $A / I$ module. Since $A / I$ is also a right Noetherian ring, we have by Goldie ([4] Theorem 2) $A / I$ is a domain and $I=P$ is a completely prime ideal of $A$. This proves (i). In the case that $M$ is not admissible, then $I$ contains $P$ properly and $F$ is the bound of $I$ in $A$. I is a prime right ideal, for, if not, then there exists elements $x, y \notin I$ such that $x A y \subseteq I$. Clearly the 
image of the right ideal $x A$ in $A / I$ namely $\overline{x A}$ is such that Ann $(\overline{x A})=P$. Hence, since $\overline{x A} \neq 0$, so $y \in P \subseteq I$ a contradiction to what we have assumed. Thus $I$ is a prime right ideal.

Next we consider localization of a noncommutative ring $A$ at a multiplicatively closed subset $S$ of any subring $R$ of $A$ which contains the identity of $R$ and which does not contain zero divisors and such that $R$ is contained in the centre of $A$. For this localization see Wangneo and Tewari ([14], p. 1579). Let $M$ be a finitely generated module over $A$ and consider the localized ring $A_{S}$ and the localized module ${ }_{S}$. Then, we have the following:

PROPOSITION 1.2. Any $A_{S}$ submodule $N$ of $M_{S}$ is of the form $W_{S}$ for some submodule $W$ of $M$.

Proof. This follows exactly as in the commutative case.

Note. We will use this fact in the sequel.

PROPOSITION 1.3. Let $A$ be a right Noetherian ring and $S$ a miltiplicatively closed subset of $R$ containing no zero divisors and containing the identity of $R$, where $R$ is a subring of the centre of $A$. If $M$ is a finitely generated right $A$ moduze, then $f^{-1}\left(\right.$ Ass. $\left.M^{1}{ }_{A^{1}}\right)=$ Ass. $\left.\left(M_{A}\right) \cap\{P \mid P \cap S=\emptyset\}\right)$, where $M^{1}=M_{S}, A^{1}=A_{S}$ and $f$ is the usual map $A \rightarrow A_{S}$.

Proof. First note that the proof of proposition (2.5) of Wangmeo and Tewari [14] can be carried over in the case of any right Noetherian ring. Together with this result and propositions 1.1 and 1.2 above the whole proof of proposition (2.6) of Wangneo and Tewari [14] carries over to yield, the required result.

Notation. Consider $B$ any right Noetherian ring with $R$ a subring of the centre of $B$ containing the identity of $B$. Let $P$ be a prime ideal of $B$. Denote by $F_{P n R}$ the set of prime ideals of $B$ lying over $P$.

DEFINITION. As on p. 1582 of Wangneo and Tewari [14], we define the support of a module $M$ over $B$ where $B$ is as in the notation above. Let $P$ be a prime ideal of $B$ and localize $M$ at $P \cap R$ in $R$ (this 
is the usual localization at the prime ideal of a commutative ring). Denote the localized module of $M$ by $M_{P \cap R}$. We say $P$ is in the support of $M$ if $M_{P \cap R} \neq 0$. We say $P$ is minimal supporting for $M$ if $P \cap R$ is minimal such that $M_{P \cap R} \neq 0$. Denote the support of $M$ by supp. $M$.

THEOREM 1.4. Let $B$ be a right Noetherian ring. Then Ass. $M \subseteq$ supp. $M$ for cony finitely generated right module $M$ over $B$, and for any minimal element $P$ of supp. $M$ there is a prime ideal in the set $F_{P \cap R}$ belonging to Ass. $M$, where $R$ is a subring of the centre of $B$ containing the identity of $B$.

Proof. With the aid of Proposition 1.1 and Proposition 1.3 above the whole proof of Theorem 2.7 of Wangneo and Tewari [14] carries over in this case.

Next, suppose that $D$ is a division ring algebraic over a subfield $K$ of its centre. It is celar that ideals of $D[X]$ lie uniquely over their contractions in $Z(D)[X]$ where $Z(D)$ is the centre of $D$. Since $Z(D)$ is algebraic over $K$, we get that $Z(D)[X]$ is integral over $K[X]$ and hence, by a well-known result from commutative algebra, we get that there is lying over, going up and incomparability between the prime ideals of $D[X]$ and $K[X]$. Invoking the Wedderburn-Artin Theorem, we can extend this result to any simple Artinian ring $S$ algebraic over a subfield $K$ of its centre. Thus we have the following proposition:

PROPOSITION 1.5. Let $S$ be a simple Artinian ring algebraic over a subfield $K$ of its centre. Then there is lying over, going up and incomparability between the prime ideals of $S[X]$ and $K[X]$.

Note. We will henceforth abbreviate lying over, going up and incomparability by 1.0, G.U., and Inc.

THEOREM 1.6. Let $A$ be a right Noetherian ming integral over a subring $R$ of its centre. Then there is $2.0, G . U$. and Inc. between the prime ideals of $R[X]$ and $A[X]$.

Proof. We will give the proof for lying over. Let $P$ be a prime ideal of $R[X]$. Let $P \cap R=P_{1}$ be a prime ideal of $R$. By Blair [2] there exists a prime ideal $Q_{1}$ of $A$ lying over $P_{1} \cdot$ Consider $\bar{A}=\frac{A}{Q_{1}}$. 
Note that $R=\frac{R}{P_{1}} \quad$ can be identified with a subring of the centre of $\bar{A}$, where $\bar{A}$ is integral over $\bar{R}$. Localize $\bar{A}$ and $\bar{R}$ at the set $S$ of regular elements of $\bar{R}$ and denote all the localized objects by suffix $S$. Clearly $\bar{R}_{S}$ is a field and, by Chamarie and Hudry ([3] corollary (1.8)), $\bar{A}_{S}$ is a simple Artinian ring. By Proposition 1.5 above, there exists a prime ideal $\bar{Q}_{S}$ of $\bar{A}_{S}[X]$ such that $\bar{P}_{S}=\bar{Q}_{S} \cap \bar{R}_{S}[X]$, where $\bar{Q}$ and $\bar{P}$ are the images of the prime ideals $Q$ and $P$ of $A$ and $R$ respectively in $\bar{A}$ and $\bar{R}$. Clearly $Q$ is a prime ideal of $A[X]$ lying over $P$ of $R[X]$.

The proofs for G.U. and Inc. are similar, and are left to the reader.

PROPOSITION 1.7. Let $M$ be a finitely generated critical right module over $A[X]$, where $A$ is a right Noetherian ring integral over a subring $R$ of its centre. Let Ann. $M=I$, and let $P$ be the assassinator of $M$, then $\frac{A[X]}{I}$ is $\frac{P}{I}$ primary.

Proof. Clearly $M$ is finitely generated, faithful and $P$-primary module over $\frac{A[X]}{I}$ (denote $\frac{A[X]}{\bar{I}}$ by $A^{1}$ ). Let $Q$ be a prime ideal associated with $A^{l}$. Then $Q=$ Ann. $M^{\perp}$ where $M^{\perp}$ is a non-zero submodule of $M$. So $Q \subseteq \frac{P}{I}$. Next, let $\frac{R[X]}{I \cap R[X]}$ be the ring denoted by $R^{1}$. Also denote the module $M$ localized at $Q \cap R^{1}$ by $M_{Q \cap R^{1}}$. Since, $M_{Q \cap R^{l}} \neq 0$, there exists a minimal prime ideal $P_{1}$ of $R^{1}$ such that $P_{1} \subseteq Q \cap R^{I}$ and $M_{P_{1}} \neq 0$ where $M_{P_{1}}$ has the usual meaning. So there exists $P_{1}^{1} \in F_{P_{1} \cap^{I}}$ such that $P_{1}^{1}$ is associated with $M$ (see Theorem 1.4). So $P_{1}{ }^{1}=\frac{P}{I}$. By what we have done above, it is apparent that $Q \cap R^{1}=\frac{P}{I} \cap R^{1}$. Since $Q \subseteq \frac{P}{I}$ and so by Inc. (Theorem 1.6) $Q=\frac{P}{I}$. So $A^{1}$ is $\frac{P}{I}$ primary.

2.

In this section we prove that every finitely generated critical module $M$ over $A[X]$, where $A$ is a Noetherian ring integral over a 
subring $R$ of its centre, is compressible.

DEFINITION. Let $M$ be a right module over a ring $B$. Then $M$ is said to be finitely connihilated over $B$ if Ann. $M$ is a finite intersection of annihilators of $M$.

PROPOSITION 2.1 (Goldie). Let $M$ be a finitely generated module over a right Noetherian ring $B$ with Ass. $M=P$. If every cyclic uniform module $W$ of $M$ is such that Ass. $\frac{B}{A n n \cdot W}=P^{1}$ and such that $W$ is $P^{1}$-torsionfree, then $M$ is finitely annihizated.

Proof. With the conditions given above on $B$ and $W$, the result follows by proposition (8) of Goldie [5].

The following proposition is useful. But before that we give a remark.

REMARK. If $P$ is a prime ideal of a right Noetherian ring $B$, then we say that the torsion theory cogenerated by $E(B / P)$ is the torsion theory at $P$. This torsion theory is the same as the torsion theory at $C(P)$ where $C(P)$ is the set of elements of $B$ regular modulo $P$. For this see Lembek [10].

PROPOSITION 2.2. Let $A$ be a might Noetherion ming integral over a subring $R$ of its centre. Let $P$ and $Q$ be prime ideals of $A[X]$ lying over a prime ideal $P_{1}$ of $R[X]$ where $P_{1}$ is of the form $q[X]$ for some prime ideal $q$ of $R$. Then the torsion theories at $P$ and $Q$ are equivalent.

Proof. By Mueller ([11], theorem (1.3)) $\frac{A[X]}{P}$ is either torsion or torsionfree with respect to the torsion theory at $Q$. If $\frac{A[X]}{P}$ is torsion in this torsion theory then by the remark above there exists an element $c$ of $A[X], c$ regular modulo $Q$ such that $c \in P$. Let us denote by $I$ the two-sided ideal generated by $c$ in $A[X]$. Then, it follows that $I \cap R[X] \subseteq P \cap R[X]=P_{1}$. Also notice that since $P_{1}=q[X]$, and $P$ and $Q$ lie over $P_{1}$, we must have $P=P^{1}[X]$ and $Q=Q^{1}[X]$ for some prime ideals $P^{1}$ and $Q^{l}$ of $A$. Clearly $P^{1} \cap R=Q^{1} \cap R=q$. Localize $A$ and $R$ at $q$ and denote these localized rings by $A_{S}$ and $R_{S}$. Denote the ideal $I$ localized at $q$ by $I_{S}$ and suppose the image 
of $c$ in $I_{S}$ is $c^{l}$. Let $c$ have the form $c=a_{0}+a_{1} X+\ldots+a_{n} X^{n}$, $a_{n} \neq 0$. It is not difficult to see that $a_{n}$ is regular modulo $Q$ and hence regular modulo $Q^{1}$. Clearly since $A$ is integral over $R$, the image of $a_{n}$ in $A_{S}$ is invertible in $A_{S}$ and so multiplying $c^{l}$ by a suitable element of $A_{S}$ gives us a monic polynomial $c_{1}$ of degree $n$ in $I_{S}$ which is regular modulo $Q_{S}$. Also since $c$ generates $I$ and $a_{n}$ is regular modulo $Q$, it follows that $c$ is a polynomial of minimal degree in $I$. Hence $c_{1}$ is a monic polynomial of minimal degree in $I_{S}$. For any $r \in A_{S}, f=\left(r c_{1}-c_{1} r\right) \in I_{S}$ and $f$ is of lower degree than $c_{1}$. Hence $f=0$. So $c_{1} \in Z\left(A_{S}\right)[X]$ and $c_{1}$ is regular modulo $Q_{S}$. Since $Z\left(A_{S}\right)[X]$ is integral over $R[X]$, it can be easily shown that there exists an element $g$ of $A_{S}[X]$ such that $0 \neq C_{1} g \in R_{S}[X]$ and $C_{1} g \notin P_{1}$. Hence $I \cap R[X] \& P_{1}$ a contradiction to what we had in first paragraph of this proposition. Hence $\frac{R}{\bar{P}}$ is torsionfree with respect to the torsion theory at $Q$. Since the above argument can be reversed, we get that the torsion theories at $P$ and $Q$ are the same.

DEFINITION. Let $B$ be a ring and $M$ a right module over $B$, such that $|M|=\alpha$. $M$ is called smooth if $|N|=\alpha$, for every non-zero submodule $N$ of $M$.

Now let $A$ be a right Noetherian ring integral over a subring $R$ of its centre. Let $M$ be a finitely generated cxitical module over $A[X]$ with Ann. $M=I$. Clearly $M$ is a finitely generated, faithful module over $\frac{A[X]}{I}$ (denote $\frac{A[X]}{I}$ by $A^{1}$ ). If $M$ is $P$-primary for some prime ideal $P$ of $A^{1}$, then $A^{l}$ is $P$-primary by proposition 1.7. Consider $R^{1}=\frac{R[X]}{I \cap R[X]}$. Let $P_{1}=P \cap R^{1}$. Localize $M, A^{1}$ and $R^{1}$ at $P_{1}$ as usual and denote these localized objects by ${ }_{H_{S}}, A_{S}^{1}$ and $R_{S}^{1}$ etc. Then we have the following proposition.

PROPOSITION 2.3. If, in the above notation, we have $\left|\frac{A_{S}^{1}}{P_{S}}\right|=1$, then $M$ is either compressible or finitely annihilated. 
Proof. Let $P=\frac{P^{I}}{I}$. If $P^{1}$ is not of the form $q[X]$ for some prime ideal $q$ of $A$, then $\frac{A[X]}{P^{1}} \approx\left(\frac{A}{P^{1} \cap A}\right)[\bar{X}]$ where $\bar{X}$ is algebraic over $\frac{A}{P^{1} \cap A}$. If $S=$ complement of $\left(P^{1} \cap R[X]\right)$ in $R[X]$ then, localizing at $S$ yields that $\left|\left(\frac{A[X]}{P^{1}}\right)_{S}\right|=0$ because $\left(\frac{A[X]}{P^{1}}\right)_{S}$ is a proper homomorphic image of $\frac{A[X]}{P^{1} \cap A[X]} S$ and $\left|\frac{A[X]}{P^{1} \cap A[X]} S\right| \leqslant 1$. So, since $\left|\frac{A^{1} S}{P_{S}}\right|=1$, we get that $P^{1}$ is of the form $q[X]$ for some prime ideal $q$ of $A$. Now, by Gordon and Robson ([6], Corollary (7.5)) there exists a prime ideal $Q_{S}^{I}$ of $(A[X])_{S}$ such that $\left|A_{S}^{1}\right|=\left|\left(\frac{A[X]}{Q^{1}}\right)_{S}\right| \cdot$ Clearly $\frac{Q^{1} S}{I_{S}}$ contracts to the prime ideal $P_{S}$ of $R_{S}^{1}$, and it is not difficult to see that $Q^{1}$ is of the form $q_{1}[X]$ for some prime ideal $q_{1}$ of $A$. So $\left|\left(\frac{A[X]}{Q^{1}}\right){ }_{S}\right|=\left|A_{S}^{1}\right|=1$. Now, if $\left|M_{S}\right|=0$, then since $M$ is uniform and hence so is $M_{S}$, it follows that $M_{S}$ is simple. Let $N$ be non-zero submodule of $M$, then $N_{S} \neq 0$ because $M$ is $P$-primary and so is $N$. So $\left(\frac{M}{N}\right)_{S}=0$ which means that there exists an element $s$ of $R^{1}$ such that $M S \subseteq N$. The multiplication by $s$ gives a monomorphism of $M$ into $N$ becasue no nonzero element of $M$ can be annihilated by $S$. So $M$ is compressible. Next consider $\left|M_{S}\right|=1$. So $\left|\frac{A^{1}}{P_{S}}\right|=\left|M_{S}\right|=1$. Let $N_{S}$ be a non-zero maximal submodule of $M_{S}$ such that $\left|N_{S}\right|=0$. Clearly $\left|\frac{M_{S}}{N_{S}}\right|=1$ and $\frac{M_{S}}{N_{S}}$ is smooth. Let $B_{S}$ be the annihilator of $\frac{M_{S}}{N_{S}}$ in $A_{S}^{1}$. so $\frac{M_{S}}{N_{S}}$ is a faithful $\frac{A^{1} S}{\bar{B}_{S}}$-module. Let $Q_{S}$ be a maximal ideal of $A^{1} S$ containing $B_{S}$. Then $Q_{S}$ clearly lies over $P_{1 S}$ of $R_{S}^{1}$ because $R_{S}^{1}$ is a local ring and $P_{1 S}$ is also a unique minimal prime ideal of $R^{1}$ for $A_{S}^{1}$ is $P_{S}$-primary (see proposition 1.7 ). So $\left|\frac{A^{1} S}{Q_{S}}\right|=1$ by the same 
argument as given in the beginning of this proposition. Hence $\left|\frac{A^{1} S}{B_{S}}\right|=1$ also. So we have that $\frac{M_{S}}{N_{S}}$ is a faithful $\frac{A^{1} S}{B_{S}}$ module and $\left|\frac{A^{1} S}{B_{S}}\right|=1$. Hence, by Gordon ([7], lemma (1.7)) $\frac{A^{1} S}{B_{S}}$ embeds in a direct sum of copies of $\frac{{ }_{S}}{N_{S}}$. So $\frac{A^{1} S}{B_{S}}$ is smooth. Let $U_{S} \subseteq \frac{M_{S}}{N_{S}}$ be a nonzero critical prime submodule of $\frac{M_{S}}{N_{S}}$ such that Ann. $U_{S}=J_{S}$ for some prime ideal $J$ of $A^{1}$. Note that $\left|U_{S}\right|=\left|\frac{M_{S}}{N_{S}}\right|=\left|\frac{A^{1}}{J_{S}}\right|=1$. Also $J=\frac{J^{1}}{\bar{I}}$ where $J^{\perp}=J_{1}[X]$ for some prime ideal $J_{1}$ of $A$. Notice also that $J^{1}$ lies over $P_{1}$. Since $\left|U_{S}\right|=\left|\frac{A^{1}}{J_{S}}\right|=1$, so $U_{S}$ is $J_{S}$ torsionfree. Now $P_{S}$ and $J_{S}$ lie over the same prime ideal of $R_{S}^{1}$. So by proposition 2.2 the torsion theories at $P_{S}$ and $J_{S}$ are the same. Hence $U_{S}$ is $P_{S}$ torsionfree also. Hence $U$ is $P$-torsionfree and since $A^{l}$ is $P$-primary we are in the situation of proposition 2.1. So $U$ is a finitely annihilated uniform module over $A^{1}$. Therefore $|U|=\left|\frac{A[X]}{A n n \cdot U}\right|=\left|\frac{A[X]}{J_{1}[X]}\right|=\left|\frac{A[X]}{q[X]}\right|>|M|$, the second and third equality follow because $J_{1}$ and $q$ lie over the same prime ideal in $R$. But this is a contradiction because $M$ is critical and $U$ is a submodule of $M / N_{i}$. So $\left|N_{S}\right| \neq 0$. Hence $M_{S}$ is smooth and by the same argument as for $\frac{M_{S}}{N_{S}}$ and $U_{S}$ above we get that $M$ is finitely annihilated over $A^{1}$ and hence over $A[X]$.

REMARK. If in the above proposition $\left|\frac{A^{1}}{P_{S}}\right|=0$, then $\left|A_{S}^{1}\right|=0$. clearly $\left|M_{S}\right|=0$ in this case and so $M$ is compressible. Combining this with the above proposition, we have the following theorem.

THEOREM 2.4. Let $A$ be a right Noetherian ring integral over a subring $R$ of its centre. If $M$ is a finitely generated critical module 
with Ass. $M=P$ then

either (i) $M$ is finitely annihilated

or

(ii) $M$ is compressible.

Also in case (i) we have that $P$ has the form $P_{1}[X]$ for some prime ideal $P_{1}$ of $A$.

In what follows we will use the concept of an affiliated bi-submodule, and affiliated series for a bimodule $B$ of a Noetherian ring $C$. One can attach to $B$ a set of primes $P_{i}$ called the affiliated set of primes of $B$ (or more strictly the affiliated set of primes of an affiliated series). For these definitions we refer the reader to stafford [13].

Before proving the main result we quote a result of Gordon ([7], Proposition (3.6)) which is actually the crucial result from which our main theorem follows.

PROPOSITION 2.5 (Gordon). Let $B$ be a Noetherian ring of Kruzz dimension $\alpha$ such that $B / N(B)$ is smooth, where $N(B)$ is the nilpotent radical of $B$.

(i) A finitely generated module $M$ is $N$-torsion (that is torsion in the torsion theory cogenerated by $E_{B}(B / N)$ ) if and only if $|M|<\alpha$.

(ii) $\frac{B}{T_{N}(B)}$ is an order in an Artinian ring if and only if every finitely generated a-critical module is compressible. ${ }_{n}^{(B)}$ is the $N$-torsion ideal of $B$ ).

THEOREM 2.6. Let $A$ be a Noetherian ring integral over a subring $R$ of its centre. Let $M$ be a finitely generated critical module over $A[X]$, then $M$ is compressible.

Proof. By Theorem 2.4 we have to consider the only case when $M$ is finitely annihilated, and the assassinator $P$ of $M$ is of the form $q[X]$ for some prime ideal $q$ of $A$. Let $I=$ Ann. $M$ in $A[X]$. Consider $A^{1}=\frac{A[X]}{I}$ and $R^{1}=\frac{R[X]}{I \cap R[X]}$ and as in proposition 2.3 let $\frac{P}{I} \cap R^{1}=J=\frac{P_{1}}{I \cap R[X]}$ for some prime ideal $P_{1}$ of $R[X]$. Localize all these objects at $P_{1}$ and denote these localized objects as usual by 
suffix $S$. Notice that $\frac{P_{1_{S}}}{(I \cap R[X])_{S}}$ is a unique maximal and minimal prime ideal of $R_{S}^{1}$ and any prime ideal of $A_{S}^{1}$ lies over $P_{1_{S}}$ and they are all finite in number. Clearly all prime ideals of $(A[X])_{S}$ containing $I$ are of the form $(Q[X])_{S}$ for some prime ideal $Q_{1}$ of $A$. Let us enumerate these prime ideals as $\left(Q_{1}[X]\right)_{S},\left(Q_{2}[X]\right)_{S}, \ldots,\left(Q_{n}[X]\right)_{S}$ for some prime ideals $Q_{1}[X], Q_{2}[X], \ldots, Q_{n}[X]$ of $A[X]$, all containing $I$ and lying over the same prime ideal $P_{1}$ of $R[X]$. Consider the nilpotent radical $N$ of $A^{I}$ and let $C(0)$ be the set of regular elements of $A^{l}$ and $C(N)$ be the set of elements of $A^{l}$ regular modulo $N$. It is well known that $C(0) \subseteq C(N)$. Conversely, let $x \in C(N)$. Denote by $N^{1}$ the image of $N$ in $A_{S}^{1}$ and by $x^{1}$ the image of $x$ in $A_{S}^{1}$. Notice that $N^{1}$ is the nilpotent radical of $A_{S}^{1}$. Consider an affiliated series $A_{S}^{1}=B_{m_{S}} \geqslant B_{m-1} \geqslant \ldots \geqslant B_{0_{S}}=(0)$ of $A_{S}^{1}$ where clearly $B_{i}$, $0 \leqslant i \leqslant m$ are bi-submodules of $A^{1}$ and $B_{i_{S}}$ is the usual localization of $B_{i}$ at $\frac{P_{1}}{I \cap R[X]}$. The affiliated primes of this series will be primes of the form $\left\{\left(\frac{P_{i}[X]}{I}\right)_{S}\right.$ for some $\left.i, 1 \leqslant i \leqslant n\right\}$. We can clearly lift this affiliated series to an affiliated series of $A^{1}$ namely $A^{1} \geqslant B_{m} \geqslant \ldots B_{0}=0$ with affiliated primes of the form $\left\{\frac{P_{i}[X]}{I}\right.$, for some $\left.i, 1 \leqslant i \leqslant n\right\}$. Now $\left|\frac{A^{1}}{x A^{1}+\frac{P_{i}[X]}{I}}\right|=\left|\frac{A[X]}{x_{1} A[X]+P_{i}[X]}\right|$ where $x=x_{1}+I$ and $A^{1}=\frac{A[X]}{I}$. Also $\left|\frac{A^{1}}{x A^{1}+\frac{P_{i}[X]}{I}}\right|<\left|\frac{A^{1}}{\frac{P_{i}[X]}{I}}\right|, 1 \leqslant i \leqslant n$. So by proposition 2.3 of stafford [13] we have that $x$ is a right regular 
element of $A^{l}$. Since $A^{l}$ has left right symmetry, the same argument gives that $x$ is left regular too. Hence $x \in C(0)$.

So $C(0)=C(N)$. Hence $A^{l}$ has an Artinian quotient ring by small's Theorem [12]. Note that $M$ is a faithful finitely annihilated module over $A^{1}$. Therefore $|M|=\left|A^{1}\right|$. Also $\left|\frac{A[X]}{P_{i}[X]}\right|=\left|\frac{A[X]}{P_{j}[X]}\right|,\{1 \leqslant i, j \leqslant n\}$ because $P_{i}$ and $P_{j}$ lie over the same prime ideal of $R[X]$. Therefore $\frac{A}{N}$ is smooth. Hence, by proposition 2.5 above of Gordon, $M$ is compressible over $A^{l}$ and hence over $A[X]$. This proves the theorem.

REMARK. Note that Theorem $1.6 \mathrm{can}$ be extended to the case of a polynomial ring in $n$-commuting indeterminates, $n \geqslant 2$, namely $A\left[X_{1}, \ldots, X_{n}\right]$. We have not been able to prove whether all finitely generated critical modules over $A\left[X_{1}, \ldots, X_{n}\right]$ are compressible. The result seems to be not known even for the ring $D\left[X_{1}, \ldots, X_{n}\right]$ where $D$ is a division ring algebraic over its centre $k$. However the result is true if $D\left(X_{1}, \ldots, X_{n}\right)=D\left[X_{1}, \ldots, X_{n}\right] \otimes_{k} k\left(X_{1}, \ldots, X_{n}\right)$ is an artinian ring, where $k\left(X_{1}, \ldots, X_{n}\right)$ is the quotient field of $k\left[X_{1}, \ldots, X_{n}\right]$. In the case of one indeterminate over $D$ this is an open problem due to Amitsur; see for example Amitsur [1]. It may be remarked that $D\left(X_{1}, \ldots, X_{n}\right)$ is artinian if and only if $D\left[X_{1}, \ldots, X_{n}\right]$ is a fully bounded Noetherian ring.

\section{References}

[1] A.s. Amitsur, "Algebras over infinite fields", Proc. Amer. Math. Soc., $7(1956), 35-48$.

[2] W.D. Blair, "Right Noetherian rings integral over their centers", J. Algebra, 27 (1973), 187-198.

[3] M. Chamarie and A. Hudry, "Anneaux Noetherians A Droit Entries sur Sous-Anneau de Leur Center", Conm. AZgebra, (2) 6 (1978), 203-222.

[4] A.W. Goldie, "The structure of prime rings under ascending chain condition", Proc. London Math. Soc., (3) 8 (1958), 589-608. 
[5] A.W. Goldie, "A survey of progress in noncommutative Noetherian rings" Ring Theory, Proc. 1978 Antwerp Conf., Lect. Notes Pure App 2. Math., 51 91979), 61-79.

[6] R. Gordon and J.C. Robson, "Krull dimension", Mem. Amer. Math. Soc., 133 (1971).

[7] R. Gordon, "Some aspects of non-commutative Noetherian rings. Lect. Notes in Math., 545 (1975).

[8] K.R. Goodearl and T.H. Lenagan, "Krull dimension of differential operator rings 111: Non commutative coefficients", Trons. Amer. Math. Soc., 275 (1983), 833-859.

[9] A.V. Jategaonker, "Jacobson's conjecture and modules over fully bounded Noetherian rings", J. Algebra, (1) 30 (1974), 103-121.

[10] J. Lambek and G. Michler, "The torsion theory at a prime ideal of a right Noetherian ring", J. Algebra, (2) 25 (1973), 364-389.

[11] B.J. Mueller, "Localization in non-commutative Noetherian rings", Conad. J. Math., 28 (1976), 600-610.

[12] L.W. Small, "Orders in Artinian rings", J. Algebra, 4 (1966), 13-41.

[13] J.T. Stafford, "On the regular elements of Noetherian rings" Ring Theory, Proc. 1978 Antwerp Conf., Lect. Notes Pure Appl. Math., 51 (1979), 257-277.

[14] C.L. Wangneo and K. Tewari, "Right Noetherian rings integral over their centers", Comm. Algebra, (15) 7 (1979), 1573-1598.

\footnotetext{
Department of Mathematics,

University of Wisconsin-Milwaukee,

Milwaukee,

Wisconsin 53201 .
} 